\title{
UEBER DIE VERERBUNG DER IRISKRYPTEN UND IHREN ZUSAMMENHANG MIT DER IRISPIGMENTIERUNG
}

\author{
von \\ Dietrich Wichmann
}

Tübingen

Unter Iriskrypten, von manchen Autoren auch Lacunen genannt, versteht man Risse und Spalten in der vorderen Grenzschicht, die teilweise auch auf das darunterliegende vordere Stromablatt übergehen. Sie variieren zahlenmässig ausserordentlich und treten in den verschiedenste Formen und Grössen auf. Im unteren Teil der Iris liegende grössere Krypten möchte Lauber (11) auf die hier liegende fetale Augenspalte zurückführen, an der sich der mesodermale Anteil der Iris (vordere Grenzschicht und die beiden Stromablätter) später und unvollkommen bilde. Durch die Iriskrause wird die Iris in den kleineren pupillaren und den grösseren ciliaren Anteil getrennt. Krypten können in beiden Teilen auftreten, in der pars pupillaris aber nur zunächst der Krause, während sie sich in der pars ciliaris fast über den ganzen Raum ausbreiten und dadurch auch dem unbewaffneten Auge leichter sichtbar werden.

Nachdem zunächst J. Weninger (19) auf die Irisstruktur als wichtiges Merkmal für die Zwillingsdiagnose hingewiesen hatte, machte es die in Deutschland stark zunehmende erbbiologische Vaterschaftsdiagnose bei Paternitätsprozessen notwendig, auch die Erblichkeit der Irisstruktur zu erforschen. Die grösste Zwillingsserie wurde von G. Jancke (8) (141 Paare) vorgelegt, Schwägerle (16) untersuchte 40 Paare, kleinere Serien stammen von Frerksen (5) mit 21 und von Eskelund (2) mit 5 Paaren. Untersuchungen an Elternpaaren und Kindern wurden nur wenige durchgeführt: Weninger (20) studierte 17, Eskelund 15 und Frerksen 4 Elternpaare mit den dazugehörigen Kindern. Es erscheint daher wünschenswert, weiteres Material zur Klärung dieses Fragenkomplexes vorzulegen.

Unser Material besteht aus Elternpaaren, die auf gerichtliches Ersuchen zur erbbiologischen Vaterschaftsbegutachtung vorgestellt wurden. Es handelt sich hierbei überwiegend um Probanden aus dem mittleren Rheinland. Man könnte einwenden, dass ein derartiges Material wegen der fraglichen Abstammungsverhältnisse für VererbungsUntersuchungen ungeeignet sei. Da aber bei derartigen Untersuchungen eine sehr umfassende Merkmalserhebung durchgeführt wird - bezüglich der Formmerkmale, halten wir uns hierbei im wesentlichen an die von Weninger und Pöch (18) sowie von Scheidt (14) vorgeschlagenen Untersuchungsschemata - dürfte die allen menschliche Urteilen anhaftende Fehlerrate hierbei nicht grösser sein als die Wahrscheinlichkeit des Auftretens illegitimer Kinder bei Familienuntersuchungen. Der morphologische Befund wird in derartigen Fällen ausserdem noch durch die Untersuchung des Hautleistensystems (Geipel, 6) und den serologischen Status ergänzt. Die Irisstruktur wird hier bei künst- 
licher seitlicher Beleuchtung mit einer Lupe inspiziert, wobei die Feinstruktur deutlich hervortritt. Fotografische Aufnahmen der Iris wurden mit einer Kleinbildkamera (ExaktaVarex mit Balgengerät und $10 \mathrm{~cm}$-Xenar) ebenfalls durchgeführt, sie sind aber besonders bei stärker pigmentierten Iriden noch nicht voll befriedrigend. Für die Augenfarbenbestimmung wurde die 20-stufige Tafel von Martin-Schultz (15) benutzt.

Wie bereits gesagt, ist der Reduktionsgrad der vorderen Grenzschicht ausserordentlich variabel. Weninger kennt drei Haupttypen: I. Vordere Grenzschicht vollständig erhalten, II. vordere Grenzschicht mehr oder weniger atrophisch, III. vordere Grenzschicht fehlend. Eskelund benutzt vier Stufen; völliges Fehlen der vorderen Grenzschicht ist ihm wie auch uns nicht begegnet. Hesch (7) teilt die Irisstruktur in strahlig, gemischt und maschig ein. Unsere Einteilung gemessen an der Kryptenbildung entspricht im wesentlichen der Eskelunds.

\begin{tabular}{c|c|c|c}
\hline \hline Weninger & Eskelund & Hesch & Wichmann \\
\hline vollständig (I) & a0 & strahlig & $\begin{array}{l}\text { Krypten fehlend } \\
\text { wenige } \\
\text { mittel } \\
\text { viele }\end{array}$ \\
atrophisch (II) & a2 & gemischt \\
maschig & a3 & - & -
\end{tabular}

Die extremen Fälle sind verhältnismässig leicht zu diagnostizieren, Schwierigkeiten bereiten aber die mittleren Grade. Da ihre Klassifizierung auch von dem Befund der gerade zur Verfügung stehenden Vergleichspersonen - also gewissermassen von der "psychologischen Situation" - abhängig sein dürfte, möchten wir im folgenden von einer weitergehenden Einteilung absehen und unser Material nur alternativ: Krypten vorhanden $(\mathrm{K})$ und Krypten fehlend $(\mathrm{k})$ einteilen. Dies ist auch durch die Zahl der von uns untersuchten Fälle geboten, da sich bei einer weitgehenden Aufteilung der Fehler der kleinen Zahl eher auswirkt. Eine Klassifizierung lässt sich wohl am besten an Fotografien vornehmen, wobei bestimmte Iriden als Vergleichsmasstab zu dienen hätten. Einen derartigen Versuch stellen die Tafeln von Hesch dar, die mir aber nicht zur Verfügung standen.

Zunächst ist $z u$ untersuchen, ob in unserer Serie Zusammenhänge zwischen der Kryptenbildung sowie Alter und Geschlecht bestehen.

Tabelle r. Kryptenbildung nach Alter und Geschlecht

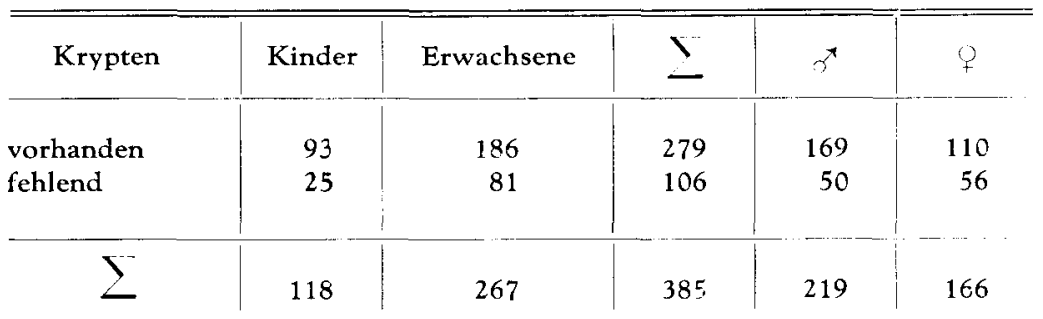


D. Wichmann: Ueber die Vererbung der Iriskrypten und ihren Zusammenhang etc.

Die Prüfung mit dem durch Yates modifizierten $\chi^{2}-$ Test zeigt, dass weder zwischen Kryptenbildung und Alter $\left(\chi^{2}=2,99\right)$ noch zwischen Kryptenbildung und Geschlecht $\left(\chi^{2}=5,09\right)$ ein gesicherter Zusammenhang besteht, da weder die Ueberschreitungswahrscheinlichkeit 0,0027 ( $3 \sigma$-Aequivalent) noch 0,01 erreicht wird (nach den Tafeln Pätaus, 12). Auch Ziegelmayer (24) fand in seiner Serie keinen Altersunterschied in der Kryptenbildung, dagegen mehr mit Krypten behaftete Männer als Frauen $\left(\chi^{2}=13,58\right\rangle$ $9,00)$.

Bei der Beurteilung der Erblichkeit der Krypten brauchen wir also in unserer Serie Alter und Geschlecht nicht zu berücksichtigen.

Tabelle 2. Die Vererbung der Kryptenbildung

$+=$ mit Krypten behaftet

$0=$ ohne Krypten

\begin{tabular}{c|c|r|r|r}
\hline \hline $\begin{array}{c}\text { Zahi d. Eltern- } \\
\text { paare }\end{array}$ & Eltern & \multicolumn{3}{|c}{ Kinder in \% } \\
\hline & & $\mathrm{n}$ & + & \multicolumn{1}{c}{0} \\
\hline 54 & $+:+$ & 58 & 91,4 & 8,6 \\
54 & $+: 0$ & 55 & 69,1 & 30,9 \\
7 & $0: 0$ & 7 & 42,9 & 57,1 \\
& & & & \\
\hline & & 120 & 78,3 & 21,7
\end{tabular}

Die Tabelle zeigt klar, dass mit abnehmender Kryptenbehaftung der Eltern auch der Anteil der Kryptenträger bei den Kindern sinkt. Die Ueberprüfung der Kinder aus den Elternkreuzungen $+:+$ und $+: 0-0: 0$ wurde wegen zu geringer Besetzung nicht berücksichtigt - ergibt, dass ein realer Unterschied zwischen den Gruppen vorliegt $\left(\chi^{2}=8,94>6,65=1 \%\right)$. In der Elternkreuzung $+:+$ kommen $z$ war weniger Kinder des entgegengesetzten Typs 0 vor als in der Elternkreuzung $0: 0$ Kinder des Typs + , was als Dominanz des 0-Typs gedeutet werden könnte. Wenn auch die Kreuzung 0:0 in unserem Material selten ist, so muss doch der Unterschied bei den Kindern als sicher $\left(\chi^{2}=8,59>6,65=1 \%\right)$ angesehen werden. Mit der Annahme einer Dominanz von 0 sind aber die Häufigkeiten bei den Kindern aus der Kreuzung +0 nicht in Einklang $z u$ bringen, denn in unserer Serie sind aus dieser Kreuzung zu wenig 0-Kinder und zu viel + Kinder entstanden. Man wird daher doch einen komplizierteren Erbgang (Polymerie) annehmen müssen. Da Kinder ihren Eltern auch oft in der Kryptenzahl ähneln, erscheint auch das Mitwirken einer Allelenreihe nicht ausgeschlossen. Auch Weninger (20) und Eskelund (2) entscheiden sich nicht für einen bestimmten Erbgang. Das Auftreten von Krypten bei den Kindern ist unabhängig davon, ob diese bei der Mutter oder beim Vater vorhanden sind. Eine zunächst enger erscheinende Korrelation zwischen Kindern und Vätern, gemessen mit dem Korrelationskoeffizienten $\mathbf{r}$, erwies sich bei Prüfung mit dem $z$-Test nicht enger als die zwischen Kindern und Müttern $(p=0.42)$.

Allen Untersuchern - Hesch, Weninger, Eskelund, Jancke, Frerksen und Lauber - 
ist auch ein Zusammenhang zwischen Augenfarbe und Kryptenbildung aufgefallen. In allen Serien treten Krypten bei dunklen Augen seltener auf als rein zufällig zu erwarten gewesen wäre, obgleich sich fast alle Untersucher technischer Hilfsmittel (Fotografie, Hornhautmikroskop, Lupe) bedienten. Der Unterschied in der Kryptenhäufigkeit ist also wohl nicht auf das Uebersehen von Krypten in stärker pigmentierten Augen zurückzuführen. Auch in unserer Serie zeigt sich ein derartiger Zusammenhang. Wie die Varianzanalyse der folgenden Tafel ergibt, ist die Streuung zwischen den Spalten wesentlich grösser als die innerhalb der Spalten $\left(Q^{2}=59,42>9,85\right)$, womit erwiesen ist, dass die Augenfarbe bei den Kryptenträgern im Durchschnitt heller ist als bei fehlenden Iriskrypten. Auch wenn unser Material weiter nach Jungen, Mädchen, Männern und Frauen aufgegliedert wird, zeigt sich in den Teilserien ein entsprechendes Ergebnis.

Tabelle 3. Zusammenhang von Krypten und Augenfarbe

\begin{tabular}{|c|c|c|c|}
\hline \multirow{2}{*}{$\begin{array}{c}\text { Augenfarbe } \\
\text { nach } \\
\text { Martin-Schultz }\end{array}$} & \multicolumn{2}{|c|}{ Krypten } & \multirow{2}{*}{ zusammen } \\
\hline & vorhanden & fehlend & \\
\hline $1 b$ & 30 & 4 & 34 \\
\hline $1 \mathrm{c}$ & 11 & 6 & 17 \\
\hline $2 a$ & 66 & 12 & 78 \\
\hline $2 b$ & 21 & 4 & 25 \\
\hline 3 & 21 & 2 & 23 \\
\hline $4 a$ & 3 & 1 & 4 \\
\hline $4 b$ & 26 & 1 & 27 \\
\hline 5 & 8 & 4 & 12 \\
\hline 6 & 29 & 5 & 34 \\
\hline 7 & 7 & 2 & 9 \\
\hline 8 & 12 & 12 & 24 \\
\hline 9 & 8 & 2 & 10 \\
\hline 10 & 19 & 15 & 34 \\
\hline 11 & 5 & 7 & 12 \\
\hline 12 & 8 & 10 & 18 \\
\hline 13 & 4 & 13 & 17 \\
\hline 14 & 1 & 5 & 6 \\
\hline 15 & -- & 1 & 1 \\
\hline zusammen & 279 & 106 & 385 \\
\hline
\end{tabular}

Die Serien von Hesch und Eskelund, letztere fehlerkritisch nicht bearbeitet, wurden von uns noch einmal durchgerechnet. Ein $\chi^{2}$ von 4,32>4,06 beweist, dass bei den Westpreussen von Hesch strahlige Struktur und braune Augen sowie blaue Augen und maschige Struktur überdurchschnittlich häufig kombiniert waren. Eskelunds Serie liess sich mit dem gleichen Test prüfen wie unsere eigene. Auch hier zeigte das $Q^{2}=11,19$; 5,16 einen einwandfreien Zusammenhang. Die Annahme einer Korrelation kann ausserdem deswegen als sicher gelten, weil verschiedene Klassifizierungen bei den Untersuchern 
D. Wichmann: Ueber die Vererbung der Iriskrypten und ihren Zusammenhang stc.

benutzt wurden: Weitergehende Aufteilung der Reduktion der vorderen Grenzschicht bei Eskelund und eine grössere Zahl von Farbstufen in unserer Serie.

Für die Varianzanalyse lässt sich zur Berechnung der Mittelwerte und Abweichungsquadrate mit Vorteil das Summenverfahren verwenden, das unseres Wissens auf Czuber (1) zurückgeht. Hierbei werden die Quadrierungen und Multiplikationen durch Summenbildungen ersetzt, wodurch rein technisch der Rechenvorgang vereinfacht wird. Benutzt man für alle Spalten der Verteilungstafel den gleichen angenommenen Mittelwert $\mathrm{A}$, so bietet sich eine Reihe von gegenseitigen Kontrollmöglichkeiten, wodurch Rechenfehler leichter behoben werden können.

Wie lässt sich dieser Zusammenhang erklären? Hesch, Lauber und Frerksen stellen nur den Zusammenhang fest. Eskelund lässt die Frage offen. Weninger und Jancke nehmen an, dass die Irisstruktur primär die Farbe beeinflusst. Grundsätzlich bestehen zwei Erklärungsmöglichkeiten.

1) Die Pigmentierung beeinflusst die Irisstruktur. In diesem Fall könnte man die Pigmentkörner in den Chromatophoren mechanisch als Verstärkung der vorderen Grenzschicht auffassen, sodass eine anlagemässig vorhandene Reduktionstendenz sich nicht auswirken kann, wodurch eine kryptenlose Iris entsteht.

2) Die Irisstruktur beeinflusst die Pigmentierung dadurch, dass durch die lückenhafte vordere Grenzschicht weniger Chromatophoren für die Aufnahme von Pigment zur Ver. fügung stehen, woraus die Aufhellung der Iris resultiert.

Wie bekannt, besteht in der Pigmentbildung in den einzelnen menschlichen Organen ein Zusammenhang. So konnte Rozprym (13) zeigen, dass bei der Farbe des Kopfhaares, der Augenbrauen, der Augenwimpern, der Iris und der Haut bestimmte Beziehungen vorliegen, da die Kombination hell bzw. dunkel gehäuft vorkommt. Die Untersuchung als Farbskale diente hier die Haarfarbentafel von Fischer-Saller - an den 153 erwachsenen Männern unseres Materials brachte bezüglich der Augenfarbe und Kopfhaarfarbe ein ähnliches Ergebnis $\left(\chi^{2}=6,6=1 \%\right.$ ). Die Gruppen hell und dunkel wurden hierbei jeweils durch das arithmetische Mittel abgegrenzt.

\begin{tabular}{c|c|c|c} 
Tabelle 4. Zusammenhang zwischen Augenfarbe und Haarfarbe \\
\hline \hline
\end{tabular}

Würde die erste Annahme stimmen, dass die Irispigmentierung die Kryptenbildung beeinflusst, dann wäre auch ein positiver Zusammenhang zwischen Haarfarbe und Kryptenbildung zu erwarten. Die statistische Analyse unseres Materials - wiederum getrennt nach Männern, Frauen, Jungen und Mädchen - ergab in allen vier Gruppen, dass kei- 
nerlei Zusammenhänge zwischen Haarfarbe und Iriskrypten nachweisbar sind. Als Beispiel bringen wir aus unserem Material eine Tabelle für die Männer. Hiernach ist also die Streuung zwischen den Spalten wesentlich kleiner als die innerhalb der Spalten.

Tabelle 5. Haarfarbe und Krypten bei Männern

\begin{tabular}{|c|c|c|c|}
\hline \multirow{2}{*}{$\begin{array}{l}\text { Haarfarbe nach } \\
\text { Fischer-Saller }\end{array}$} & \multicolumn{2}{|c|}{ Krypten } & \multirow{2}{*}{ zusammen } \\
\hline & vorhanden & fehlend & \\
\hline A & 1 & - & 1 \\
\hline B & - & - & - \\
\hline $\mathrm{C}$ & - & - & $-\rightarrow$ \\
\hline $\mathrm{D}$ & - & -- & $\ldots$ \\
\hline $\mathrm{E}$ & - & - & $\ldots$ \\
\hline $\mathrm{F}$ & -- & -- & - \\
\hline G & 2 & -- & 2 \\
\hline $\mathrm{H}$ & 1 & 1 & 2 \\
\hline $\mathbf{J}$ & -- & 1 & 1 \\
\hline$K$ & 1 & -- & 1 \\
\hline $\mathbf{L}$ & 3 & - & 3 \\
\hline M & 2 & 1 & 3 \\
\hline $\mathrm{N}$ & 4 & 1 & 5 \\
\hline $\mathrm{O}$ & 3 & 2 & 5 \\
\hline$P$ & 9 & 2 & 11 \\
\hline$Q$ & 1 & 2 & 3 \\
\hline $\mathrm{R}$ & 14 & 3 & 17 \\
\hline$S$ & 9 & 2 & 11 \\
\hline $\mathrm{T}$ & 10 & 4 & 14 \\
\hline $\mathrm{U}$ & 23 & 5 & 28 \\
\hline $\mathrm{V}$ & 13 & 4 & 17 \\
\hline W & 14 & 7 & 21 \\
\hline $\mathrm{X}$ & 6 & 3 & 9 \\
\hline \multirow[t]{2}{*}{ zusammen } & 116 & 38 & 154 \\
\hline & $\begin{array}{l}\text { mer } Q= \\
\text { gen } Q= \\
\text { gen } Q= \\
\text { dehen } Q=\end{array}$ & $\begin{array}{c}<295 \\
293 \\
<3,14 \\
<295\end{array}$ & \\
\hline
\end{tabular}

In keinem Fall — auch bei den Jungen nicht — wird auch nur die $2 \sigma$-Grenze erreicht. Da für die erste Annahme, dass die Pigmentbildung die Reduktion der vorderen Grenzschicht beeinflusst, aus unserem Material kein Beweis zu erbringen ist, verbleibt nur die zweite Hypothese.

Diese lässt sich auch durch entwicklungsgeschichtliche Gründe stützen. So konnte Lauber (10) zeigen, dass bereits bei Feten im 8. Monat sich Krypten mit der Rückbildung der Pupillar-Membran in der pars pupillaris entwickeln, die in der pars ciliaris an der 
D. Wichmann: Ueber die Vererbung der Iriskrypten und ihren Zusammenhang etc.

Iriswurzel gelegenen entwickeln sich mit der Rückbildung des Lig. pectinatum im 9. Monat. Auch Kolmer (9) konnte - im Gegensatz zu Weninger (20) — beim Neugeborenen Krypten nachweisen. Bei der Geburt zeigen die europäischen Kinder eine graublaue Irisfarbe. Die Irispigmentierung tritt erst im Laufe der ersten postfetalen Monate ein. Jedoch berichtet Vogt (17), dass er bulbi eines Feten im 8. Monat mit tiefbrauner Iris gesehen habe, betont aber, dass dies eine Ausname von der Regel sei.

\section{Zusammenfassung}

Es wird ein grösseres Familienmaterial über die Vererbung der Iriskrypten vorgelegt. Die statistische Analyse ergibt mit Wahrscheinlichkeit keinen monomeren Erbgang. Die auch von früheren Untersuchungen bekannten Zusammenhänge zwischen Iriskrypten und Irispigmentierung liessen sich bestätigen. Der Zusammenhang wird dadurch zu erklären sein, dass die Iriskrypten - entwicklungsgeschichtlich früher auftretend - in der vorderen Grenzschicht weniger Chromatophoren für die Pigmentbildung übrig lassen. Daraus ergibt sich, dass die Irisfarbe, wie sie beim Vergleich mit einer Augenfarbentafel festgestellt wird, wohl kein monomeres Erbmerkmal ist. Es scheint auch bedenklich, wenn Irisfarbe und Irisstruktur im polysymptomatischen Aehnlichkeitsvergleich - etwa bei der Eiigkeits-Diagnose in der Zwillingsforschung oder bei der Vaterschaftsdiagnose nebeneinander verwendet werden, da die logische Voraussetzung des polysymptomatischen Aehnlichkeitsvergleiches eben die Unabhängigkeit der miteinander verknüpften Teilindizien ist. Dies gilt in jedem Fall, einerlei ob man für die kritische Ueberprüfung derartiger Untersuchungen statistische Methoden wie z. B. die Essen-Möller-Formel $(3,4)$ benutzt (Wichmann 22,23 ) oder nicht.

\section{Literaturverzeichnis}

1. Czuber, E., Die statistischen Forschungsmethoden. Wien 1927.

2. Eskelund, V., Structural Variations of the human iris and their heredity. Kopenhagen $u$. London 1938.

3. Essen-Möller, E., Mitt. Anthr. Ges. Wien, 68, 1938.

4. - Hereditas 27, 1941.

5. Frerksen, E., Zeitschr. Anatomie u. Entwickl. gesch. 109, 1939.

6. GeIPEL, G., Anleitung zur erbbiologischen Beurteilung der Finger-und Handleisten. München 1935.

7. Неsch, M., Verh. Ges. phys. Anthr. 5, 1931.

8. JANCKe, G., Graefes Archiv f. Ophthal. 142, 1941.

9. Kolmer, Zitiert nach LaUber 11.

10. Lauber, H., Graefes Archiv f. Ophthal. 68, 1908.

11. - Die Regenbogenhaut, in W. v. Möllendorff, Handb. d. mikr. Anat. III, 2, Berlin 1936.

12. PÄtau, K., Ztschr. indukt. Abst. u. Vererbl. 80, 1942.

13. Rozprym, F., J. Roy. Anthr. Inst. of Great Britain and Ireland 54, 1934.

14. ScheIDT, W., Physiognomische Studien an niedersächsichen u. oberschwäbischen Landbevölkerungen. Jena 1931.

15. Schultz, B. K., Anthr. Anz. 6, 1930.

16. Schwägerle, F., Zeitschr. menschl. Vererb. u. Konst. lehre, 22, 1938.

17. Vogt, A., Lehrbuch u. Atlas der Spaltlampenmikroskopie des lebenden Auges. 3, Stuttgart 1942.

18. Weninger, J., u. H. Pöch, Mitt. Anthr. Ges. Wien 55, 1924. 
19. Weninger, J., Mitt. Anthr. Ges. Wien, 62, 1932.

20. - Zeitsch. Morph. Anthr. 34, 1934.

21. - Die anthropologischen Methoden der menschlichen Erbforschung. In Handb. d. Erbbiol. d. Menschen, herausgegeb. v. G. Just, 2, 1940.

22. WichmanN, D., Homo 2, 1951.

23, - Zeitschr. Morph. Anthr. 44, 1952.

24. Ziegelmayer, G., Die Heilkunst, 64, 1951.

\section{RIASSUNTO}

Si presenta un discreto materiale familiare per studiare l'eredità delle cripte dell'iride, e ne risulta che l'eredità probabilmente non è monoibrida. Si confermano i rapporti tra cripte e pigmentazione dell'iride, noti anche $\mathrm{da}$ ricerche precedenti. Si può spiegare il rapporto dal fatto che le cripte dell'iride - che compaiono già prima durante lo sviluppo embriologico - lasciano nello strato limitante anteriore meno cromatofori per la formazione di pigmento. Ne risulta che il colore dell'iride, determinato confrontandolo con una tavola per i colori dell'occhio non dovrebbe essere un carattere ereditario monoibrido. È dubbio, persino, se è possibile adoperare contemporaneamente il colore e la struttura dell'iride in funzione di un confronto di rassomiglianza polisintomatica - come per la diagnosi di gemelli monovulari o per la diagnosi della paternità - giacchè la premessa logica per un confronto polisintomatico di somiglianza viene infatti costituita dalla indipendenza degli indizi parziali connessi tra di loro. Ciò vale in qualunque caso, sia che per l'esame critico di tali ricerche si adoperino metodi statistici, come per es. la formula di Essen-Möller (3, 4) (Wichmann 22, 23) o no.

\section{RÉSUMÉ}

SUMMARY

On dispose de données familiales assez abondantes pour étudier les cryptes de l'iris, et il en résulte que l'hérédité n'est probablement pas monohybride. Cela se confirme par les relations connues également par des recherches précédentes. On peut expliquer la relation par le fait que les cryptes de l'iris (qui apparaissent déjà auparavant durant le développement embryologique) laissent dans la couche voisine antérieure moins de chromatophores pour la formation du pigment. Il en résulte que la couleur de l'iris déterminée en la confrontant avec un tableau des couleurs de l'œil ne devrait pas être un signe héréditaire monohybride.

Le doute subiste même si on emploie en même temps la couleur et la structure de l'iris pour un essai de ressemblance polysymptomatique (comme pour le diagnostic de jumeaux monovulaires ou pour le diagnostic de la paternité), attendu que la condition logique pour une confrontation polysymptomatique est de fait constituée par l'indépendance des indices partiels reliés entre eux. Ceci est valable dans tous les cas, soit que pour l'examen critique de telles recherches on emploie des méthodes statistiques, comme par exemple la formule de Essen-Möller (3, 4) (Wichman 22, 23), ou non.
A certain amount of material is provided for studying the heredity of fissures of the iris, and it seems that such a heredity is probably not of monohybrid nature. The relationship between fissures and pigmentation of the iris has been confirmed and noted also by earlier researches. This connection may be explained by the fact that fissures of the iris-appearing even earlier, during embriological developmentleave fewer chromatophores in the stratum limitans anterior for the formation of pigment. It thus follows that the colour of the iris, which is determined by comparing it with table of colours of the eye, would not be of monohybrid heredity. It is doubtful even when both colour and structure of the iris are contemporaneously studied in order to realise a polysymptomatic resemblance-as for the diagnosis of monovular twins of the diagnostic of paternity-since the logical basis for establishing a polysymptomatic comparison of resemblance is, in fact, given by the independance of partial signs connected one with another. This goes for all cases, whether or no statistical methods are used for the critical examination of such researches, such as, for example, Essen-Möller's formula $(3,4)$ (Wichmann 22, 23). 